\title{
PRISMA: A NEW SPACE MISSION FOR STELLAR PHYSICS
}

\section{T. APPOURCHAUX}

ESA/ESTEC, P.O.Box 299, 2200AG Noordwijk, The Netherlands

D. GOUGH

Institute of Astronomy, Madingley Road, Cambridge CB3 OHA, England

P. HYOYNG

SRON, Sorbonnelaan 2, 3584 CA Utrecht, The Netherlands

C. CATALA

DESPA - Observatoire de Paris-Meudon, 92100 Meudon, France

\section{S. FRANDSEN}

Aarhus Universiteit, Langelandsgade, 8000 Aarhus C, Denmark

C. FRÖHLICH

PMOD/WRC, POB 173, 7260 Davos Dorf, Switzerland

\section{A. JONES}

Aarhus Universiteit, Langelandsgade, 8000 Aarhus C, Denmark

P. LEMAIRE

IAS, BP 10, 91371 Verrieres-le-Buisson Cedex, France

G. TONDELLO

Universita di Padova, Via Gradenigo 6A, 35100 Padova, Italy

W. W. WEISS

Institute für Astronomie, Türkenschanzstraße 17, 1180 Wien, Austria

\section{INTRODUCTION}

PRISMA (Probing Rotation and Interior of Stars: Microvariability and Activity) is a new space mission of the European Space Agency. PRISMA is currently in a Phase A study with 3 other competitors. PRISMA is the only ESA-only mission amongst those four and only one mission will be selected in Spring 1993 to become a real space mission.

The goal of the Phase A study is to determine whether the payload of PRISMA can be accommodated on a second unit of the X-ray Multi-Mirror (XMM) bus; and whether the budget of the PRISMA mission can be kept below 265 MAU ('88 Economic conditions). The XMM mission is an approved cornerstone and is in a Phase A together with PRISMA. 
This paper will mainly focus on the scientific objectives that can be achieved with PRISMA. Figure I gives an overview of the PRISMA scientific objectives. The scientific objectives can be classified into three areas of interest:

- Stellar structure and evolution

- Stellar atmospheres and magnetic fields

- Stellar dynamics and stellar dynamos

These three subjects are developed in section 1. A short overview of the observing programme is also given in section 2 . The payload is outlined in section 3 . And we conclude on the future of the PRISMA mission in the epilogue.

\section{SCIENTIFIC OBJECTIVES}

\section{Stellar structure and evolution}

The impressive success of the theory of the structure and evolution of stars in reproducing classical astronomical observations of stellar surfaces has convincingly demonstrated that the theory is more-or-less right. We can therefore safely presume that at least the gross properties of stars have been correctly explained. However, there remain apparently minor discrepancies between theory and observation which might be symptomatic of serious flaws in some quite important aspects of our modelling of stellar interiors, yet which hardly influence the surface properties at all. Only by probing beneath the stellar surface can those superficially subtle aspects of the internal structure be measured.

It is the objective of PRISMA to measure such properties. Although stars are optically opaque, they are acoustically transparent. Therefore, by measuring and analysing the frequencies of acoustic oscillations, a wealth of qualitatively new information about the internal structure of stars can be acquired. This information provides us with not only measures of gross properties of stars, such as mean density, but also with data from which one can ascertain important detailed features of the internal structure that control evolution.

Acoustic modes are confined within a cavity of propagation whose upper boundary is very near the surface of the star. Consequently the modes can be detected by observing modulations of the photosphere. PRISMA aims at measuring variations in the radiative intensity induced by the oscillations. Those variations can be analysed to determine the characteristic frequencies of modes of low degree, all of which penetrate deeply into the star, some reaching even to the very centre.

Different aspects of the oscillation spectrum manifest different properties of the stellar interior. For example, the mean frequency separation between modes of differing order but the same degree, the easiest of all seismic signatures to measure, essentially determines the mean density of the star, which is proportional to $M / R^{3}$. When coupled with a spectroscopic determination of surface gravity, $G M / R^{2}$, the mass $M$ and radius $R$ of the star can then be separately determined. The mean frequency displacement between spectra of modes of different degree measures sound-speed variation in the energy-generating core, and can thus be used as a direct signature of chemical inhomogeneity produced by 


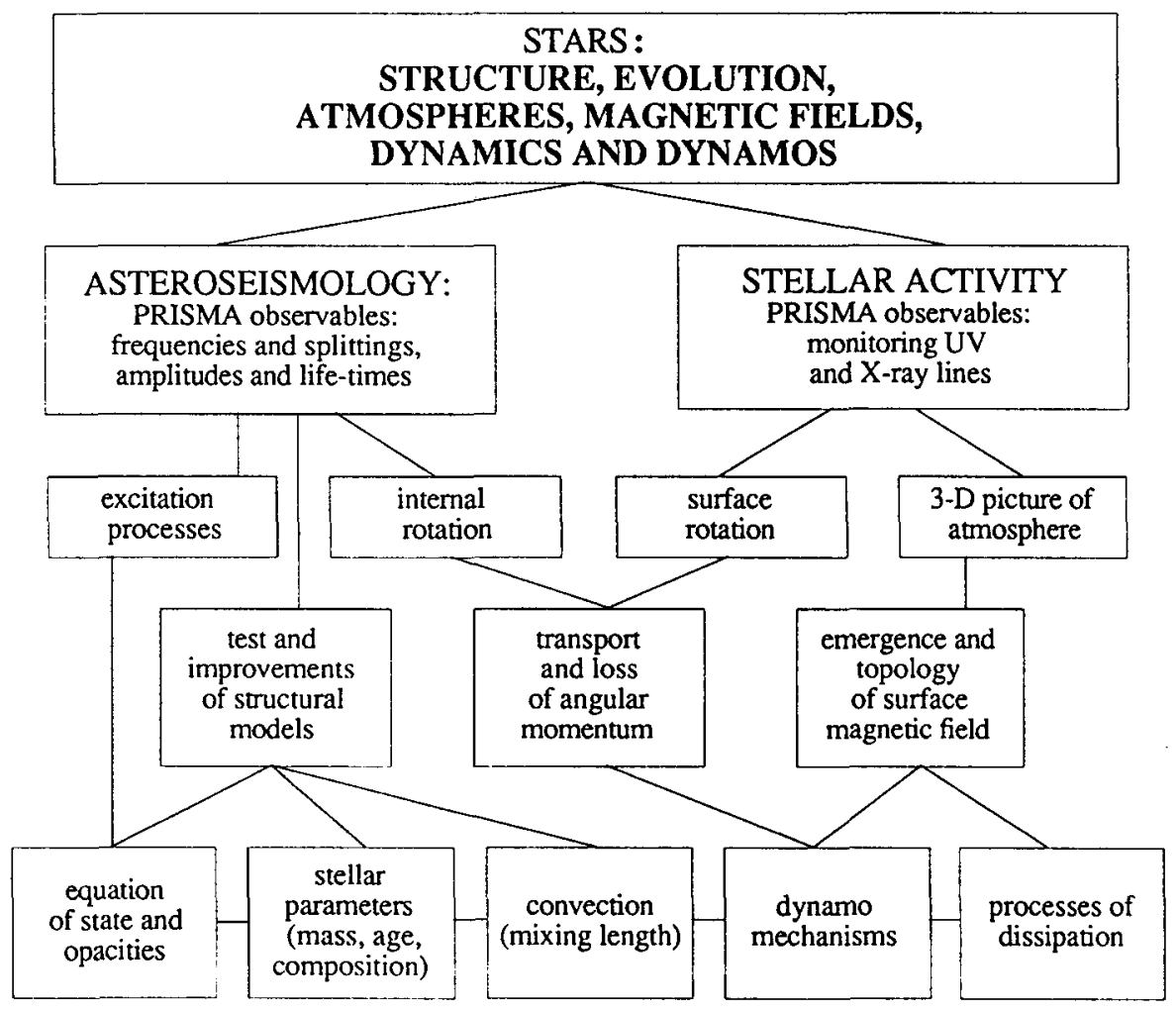

FIGURE I Scientific objectives of PRISMA 
the nuclear reactions. The degree of inhomogeneity is a measure of age. But more important still are the sophisticated analyses that provide measurements of the variation of density throughout the core, or the degree of overshoot at the base of the envelope of a late-type main-sequence star. Both those diagnostics are important to our understanding of the redistribution of chemical elements, a knowledge of which is essential for a proper appreciation of the implications of chemical abundance analyses of stellar atmospheres based on classical spectroscopic data. Material redistribution influences the chemical and structural evolution of the star, and is therefore a crucial element in the comparison of theory with observation in the H-R diagram.

An important component of the PRISMA investigation is the calibration of theoretical models of open clusters, aided by data from HIPPARCOS. Not only will this permit a determination of the cosmologically important internal stellar helium abundance, but it will also yield direct measures of the ages of the clusters, and thereby improve our ability to diagnose evolutionary properties of stars. The information so acquired will make our determinations of the ages of globular clusters, inferred through theoretical modelling, more secure, even though stars in those clusters will not be directly accessible to PRISMA. That will strengthen the limits that can be placed on the age of the Galaxy.

\section{Stellar atmospheres and magnetic fields}

It is now accepted that stellar activity is the manifestation of the interaction of magnetic fields with the plasma in the atmospheres of stars. The magnetic field is believed to be generated by a magnetic dynamo seated in the convecting envelope. The basis for this picture is the Sun, where buoyant magnetic fields produced by the dynamo are seen to rise through the photosphere to form bipolar active regions. The polarity and latitude of emergence of active regions exhibit a cyclic behaviour with a period of 22 years, the solar cycle. The entire solar surface is covered with the decay products of old active regions. Consequently, the surface magnetic flux is organised in flux tubes and highly fragmented. Flux tubes guide energy from subphotospheric layers, which heats the outer atmosphere to temperatures of $10^{6} \mathrm{~K}$. As the photospheric flow field carries the magnetic field along, there ensues a hierarchy of magnetic instabilities, the most impressive of which is a large solar flare. The theoretical basis for these phenomena is much less secure than the observational evidence. Despite vigorous efforts, we do not understand how the solar dynamo works, although differential rotation and helical convection are known to be important ingredients. Starting from a poloidal field the former generates a strong toroidal field, which is converted in turn into a poloidal field by the helical convection (the $\alpha$ effect), and the result is a periodic dynamo. But the details of this process, and in particular the many subtle nonlinear interactions remain to be filled in. There is an urgent need to test theories of magnetic dissipation and of the magnetic dynamo with a larger sample of stars than just our Sun. This has been the drive behind the 'Solar Stellar Connection', which field is now about ten years old, and which has yielded ample evidence that solar-type magnetic activity exists in all rotating stars sufficiently cool to have a convective envelope. Such a test is therefore well possible but requires a concerted effort.

A major aim of PRISMA is to provide the necessary observations of stellar 
activity in a systematic fashion, such that the H-R diagram is well covered. As stars rotate while being observed by the XUV, UV and optical monitors of PRISMA, the modulation in the signals caused by the irregular distribution of magnetic fields over the stellar surface can be used to determine the surface rotation and to construct a crude magnetic image of the surface of the star. Either by Doppler imaging using spectral lines, or modulation imaging using broad-band signals (such as provided by white- light and XUV monitors), the combined signals will allow plage mapping, revealing the spot contents of plages on stars, and also the emission scale height of the stellar coronae. With this information we can study the mechanisms heating the outer atmospheres of cool stars in much more detail than in present-day theories in which results depend on too many independent variables.

\section{Stellar dynamics and stellar dynamos}

The cross-fertilization of stellar activity and asteroseismology is particularly evident for the problem of the internal dynamics and magnetic dynamos of stars. An important goal of the PRISMA mission is the determination of the mean internal angular velocities of stars. Together with measures of the surface rotation, that will provide information about the spatial variation of the rotation in the interior, which is crucial to our understanding of the distribution of angular momentum. Temporal variations can be inferred from observations of cluster stars, for which we shall have improved seismic age determinations. Small acoustic frequency variations associated with activity have been observed on the Sun, and appear to be due to magnetically induced modifications to the subphotospheric outer reflecting surface of the acoustic cavity. We therefore anticipate much greater variations in more active stars. Rotation, differential rotation, and latitude distribution of stellar activity, combined with information on the depth of the convection zone (from refined stellar models) and on the cycle period (from ground-based measurements) are the essential ingredients for modelling stellar dynamos. The object is to derive scaling laws and to test these with the help of a sufficiently large sample of stars. An example of an interesting class of objects to test dynamo theories are early $F$ stars close to the granulation boundary. These stars have shallow convection zones, and are expected to have substantial radial differential rotation due to efficient magnetic breaking. At the same time, these stars should feature relatively strong global oscillations, allowing a good determination of their internal structure and rotation. For stars with short magnetic cycles there is even the perspective that we may observe different phases of the cycle.

\section{OBSERVING PROGRAMME}

To provide powerful tests for the models of stellar structure and evolution, and to constrain theories of angular momentum transport and of magnetic field generation by dynamo action, several requirements must be imposed on the choice and number of targets to be observed by PRISMA.

In the first place, the targets must correctly sample the stellar parameter space (mass, temperature, age). Secondly, it is necessary to observe stars with different rotation rates. It can easily be seen that a significant sampling of the 
(spectral type - luminosity class - rotation rate) space cannot be reached with a number of targets smaller than about 100 . To be able to observe more than 100 targets in a two-year mission, with exposure times of 1 month per target, the strategy is adopted in which several stars are observed simultaneously.

A better test of stellar structure models can be achieved if one or several basic stellar parameters are known independently. This is why a very high priority must be given to possible targets for which independent information is available, or for which additional constraints can be placed on the models:

- Members of open clusters, for which a good estimate of the age is available.

- Members of some binary systems, for which the masses, and sometimes the radii, are well known.

- Stars observed by HIPPARCOS, for which we have a good determination of the absolute luminosity.

- Stars observed by IUE and ROSAT

- Stars observed in the Mount Wilson Call program

Besides these prime targets which will drive the instrument and strategy definition, the scientific return of the mission will be maximized if a sample of very important targets are included: these are the classical pulsating variables, such as $\delta$ Scuti, RR Lyrae, Cepheids, $\lambda$ Boötis, and also rapidly oscillating Ap stars, Am stars and Maia variables. These targets should not drive the major requirements of the mission, but the philosophy would be to observe them with PRISMA whenever possible.

\section{MODEL PAYLOAD AND OBSERVABLES}

The model payload for PRISMA comprises 4 instruments. There are 2 photometric instruments:

- A Large Photometer (LP), with an aperture equivalent to $41 \mathrm{~cm}$ diameter, has a $1.5^{\circ} \times 1.5^{\circ}$ field of view. The LP uses a 3-mirror off-axis design to reduce the effects of straylight. It collects simultaneously the broad band visible flux of stars brighter than magnitude 8 to detect micromagnitude flux variations.

- A Small Photometer (SP), with an aperture equivalent to $14 \mathrm{~cm}$ diameter, has a $3^{\circ} \times 3^{\circ}$ field of view. It has the same purpose and design as the LP except that micromagnitude flux variations will be detected on stars brighter than 6th magnitude. An internal mirror allows a wide selection of fields offset from the line of sight of the other instruments.

The observables of the Large and Small Photometers are summarized in Table I. And there are 2 spectroscopic instruments:

- An Ultra Violet Spectrometer (UVS), optically aligned with the Large Photometer, has a $60 \times 45 \mathrm{~cm}$ aperture and covers the $120-285 \mathrm{~nm}$ wavelength range that includes chromospheric and transition region lines. A 
TABLE I PRISMA observables: Photometric segment

\begin{tabular}{|c|c|c|c|}
\hline Observables & Range & Accuracy & Scientific keywords \\
\hline Frequencies & $0.02 \mathrm{mHz}-0.1 \mathrm{~Hz}$ & $0.1 \mu \mathrm{Hz}$ & $\begin{array}{l}\text { Stellar structure, } g \text { modes, } \\
\text { He content, neutrino physics }\end{array}$ \\
\hline Amplitudes & $10^{-6}-10^{-1}$ & $10^{-7}-10^{-5}$ & $\begin{array}{l}\text { Excitation, stellar spots } \\
\text { and plages, non-linear } \\
\text { pulsators?, convection }\end{array}$ \\
\hline Linewidths & $1-10 \mu \mathrm{Hz}$ & $0.05 \mu \mathrm{Hz}$ & $\begin{array}{l}\text { Excitation, convection, } \\
\text { surface structure }\end{array}$ \\
\hline $\begin{array}{l}\text { Frequency } \\
\text { separations }\end{array}$ & $>0.3 \mu \mathrm{Hz}$ & $0.1 \mu \mathrm{Hz}$ & $\begin{array}{l}\text { Stellar parameters, stellar } \\
\text { structure, rotation, } \\
\text { magnetic fields }\end{array}$ \\
\hline
\end{tabular}

high-resolution cross-dispersed echelle system allows observation of one star at a time in a $1.5^{\circ} \times 1.5^{\circ}$ field of view (Resolution 0.004-0.01 nm).

- An eXtreme Ultra Violet Telescope (XUVT) images the same $1.5^{\circ} \times 1.5^{\circ}$ field as the Large Photometer, in 2 bands sensitive to coronal temperatures (possibly centered at $14 \mathrm{~nm}$ and $17 \mathrm{~nm}$ ).

The observables of the UV Spectrometer and XUV Telescope are summarized in Table II.

TABLE II PRISMA observables: Spectroscopic segment

\begin{tabular}{|c|c|c|c|}
\hline Observables & Range & Accuracy & Scientific keywords \\
\hline UV flux & $10^{-13}-10^{-17} \mathrm{~W} \mathrm{~m}^{-2}$ & $5 \%$ & $3 \mathrm{D}$ pictures \\
\hline UV line-profiles & $120-285 \mathrm{~nm}$ & $\begin{array}{c}0.004-0.01 \mathrm{~nm} \\
\text { (resolution) }\end{array}$ & Doppler imaging \\
\hline X-ray flux & $10^{-14}-10^{-17} \mathrm{~W} \mathrm{~m}^{-2}$ & $20 \%$ & Corona, flares \\
\hline
\end{tabular}

A more detailed description of the PRISMA payload can be found elsewhere in these proceedings (Jones, 1992). 


\section{$\underline{\text { EPILOGUE }}$}

The combination of asteroseismology and stellar activity is a wholly new concept in stellar investigation. Helioseismology has already reaped enormous rewards, and has led to a much more secure and precise knowledge of the internal structure and rotation of the Sun. This has led in turn to important consequences for solar dynamo models. The PRISMA mission aims to extend that understanding to other stars. It has been demonstrated already by helioseismology and its theoretical asteroseismic extensions that from the low-degree modes that will be available we can increase in our knowledge of other stars really significantly, particularly in their cores, in which thermonuclear energy is being generated. Indeed, the PRISMA mission can do that for a range of stars. Therefore, not only will that provide us simply with a greater knowledge of the structures of those stars, but, taken together, the sum of the information on stellar activity and seismology will substantially improve our knowledge of the mechanisms of magnetic activity, magnetic braking and especially stellar evolution, and with that, significantly increase our understanding of the nature of the Universe.

The future for the PRISMA mission is bright. This mission has no precursor and it will return new science. Not only will we be able to understand the insides of the stars, but in the long term it will bring us a better comprehension of the outsides of the stars. We may quote Malcolm Longair who said in his opening talk:

In my view, in the case of projects such as PRISMA, it is not a question of 'If it will fly' but rather 'When it will fly'. The scientific significance seems to me unassailable.

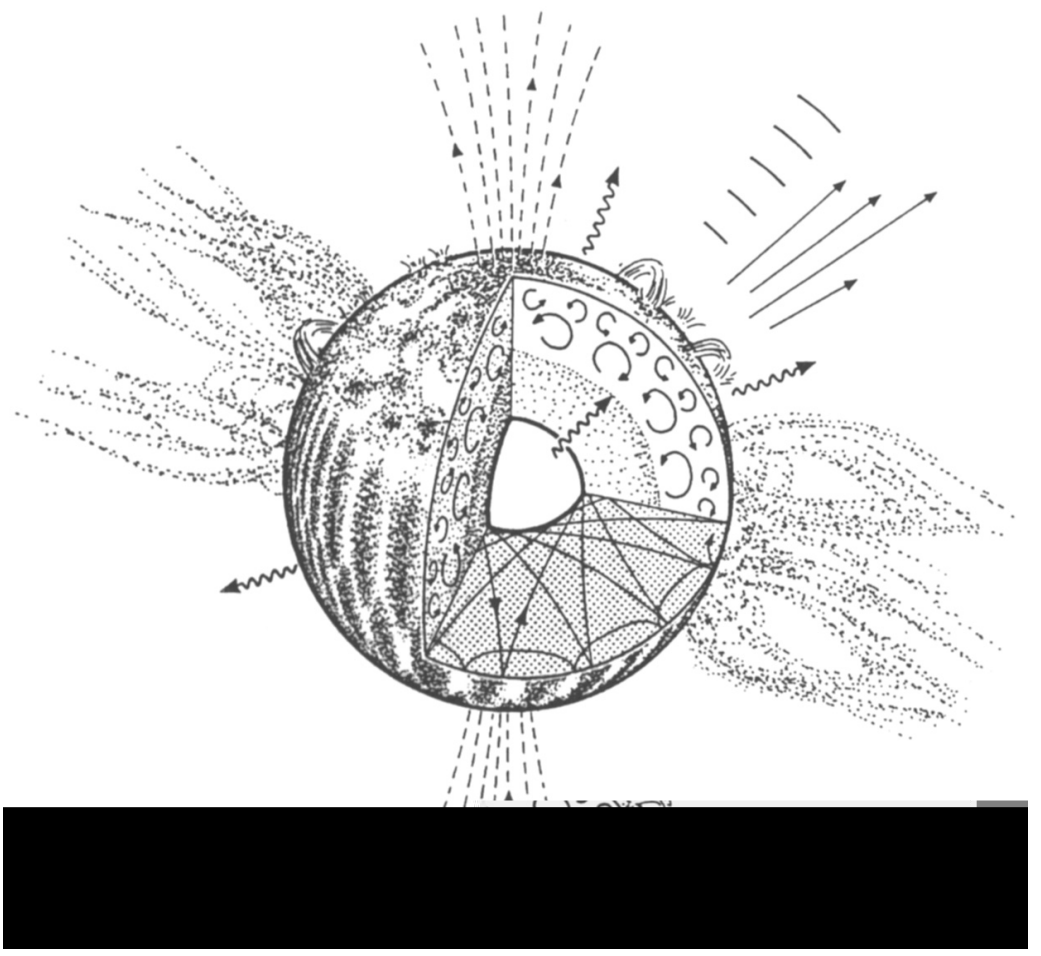

